\title{
Health Care Transition in Youth With Type 1 Diabetes
}

and an A1C $>9 \%$ : Qualitative Analysis of

Pre-Transition Perspectives

\author{
Elise Schlissel Tremblay, ${ }^{1}$ Jessica Ruiz, ${ }^{2}$ Tess Buccigrosso, ${ }^{1}$ Taylor Dean, ${ }^{1}$ and Katharine Garvey ${ }^{1}$ \\ ${ }^{1}$ Department of Pediatrics, Division of Endocrinology, Boston Children's Hospital, Boston, MA; ${ }^{2}$ Boston Combined Residency Program, Boston \\ Children's Hospital, Boston, MA
}

OBJECTIVE I To explore expectations for transition to adult care and experiences with transition planning among adolescents and young adults with type 1 diabetes and an A1C $>9 \%$ at a tertiary care U.S. pediatric center.

METHODS I We conducted semi-structured interviews in a purposive sample of patients 14-23 years of age who had had type 1 diabetes for at least 1 year and had an A1C >9\%. A multidisciplinary team conducted iterative thematic analysis with deductive and inductive coding aided by NVivo software.

RESULTS I Fourteen subjects participated (nine adolescents and five young adults, mean age $17.1 \pm 3.2$ years, $57 \%$ male, 79\% Caucasian, 14\% Hispanic, diabetes duration $8.2 \pm 4.6$ years, mean A1C $10.0 \pm 0.8 \%$ for adolescents and $10.1 \pm 0.7 \%$ for young adults). Qualitative analysis yielded four key themes. The first was lack of formal preparation; participants of all ages demonstrated a lack of preparation for transition and ignorance about the process, describing it as coming "out of the blue." The second was a desire for delayed and gradual transition; participants wanted to defer being "serious" about transition to a later/uncertain date, with a preference to "wait until I'm older" among all ages. Participants described ideal transition as a gradual process, taking place "a little at a time." The third was attachment to pediatric providers; participants demonstrated a nearly universal attachment to and "familiarity" with their pediatric diabetes care providers and expressed worries about an "uncomfortable" transition to adult providers. The fourth was concern about an impersonal adult care setting: participants perceived adult care as "formal," "scarier," and "tougher," with increased criticism about poor control; participants expressed fear that adult providers would not "know me" or appreciate "my diabetes journey."

CONCLUSION I We demonstrated a lack of transition preparation and anxiety about transition and adult care among youth with type 1 diabetes and elevated A1C. Our results may help guide early, iterative pediatric transition counseling, with a special focus on addressing attachment and fears about adult diabetes care.

Health care transition is defined as "the planned, purposeful movement of young adults from child-centered to adult-oriented health-care systems" (I). On the cusp of what psychologist J.J. Arnett describes as an "emerging adulthood," young adults face a myriad of competing needs and new responsibilities, making it a uniquely challenging time (2). There is broad expert consensus that transitioning young adults with chronic illness are at risk for interruptions in care and adverse health outcomes (3). Each chronic childhood disease presents its own unique set of challenges to the transition paradigm, making it essential that any general model for transition of care be adapted to each particular disease process.

Type I diabetes places intense self-management demands on adolescents and young adults and poses significant care coordination barriers for both adult and pediatric diabetes care providers. Transition challenges documented in the literature include gaps between pediatric and adult care, inadequate preparation by pediatric providers (i.e., lack of transition-related preparation counseling and explicit coordination of the transition process), increased post-transition hospitalizations, and general dissatisfaction with the experience

Corresponding author: Elise Schlissel Tremblay, elise.tremblay@childrens.harvard.edu

This article contains supplementary material online at https://doi.org/10.2337/figshare.12512750.

https://doi.org/10.2337/ds20-0011

(C)2020 by the American Diabetes Association. Readers may use this article as long as the work is properly cited, the use is educational and not for profit, and the work is not altered. More information is available at https://www.diabetesjournals.org/content/license. 
(4-II). An ideal transition of care would include standardized assessment of patient readiness and measurement of patient outcomes, neither of which are systematically incorporated in practice $(12,13)$.

Multiple professional organizations have written formal transition guidelines; however, the implementation of these guidelines is generally lacking and varies largely by country, region, diabetes program, and even by provider within programs (I4,15). The American Academy of Pediatrics (AAP) updated guidelines on care transition from 2018 highlight specific domains of responsibility for pediatric and adult providers in the transition process and provide additional guidance for transitions for youth with special health care needs (i.e., type I diabetes), including the need for additional support such as care coordination services (3). The American Diabetes Association (ADA), in its 2019 update, highlights the need for pediatric providers to initiate transition preparation in early adolescence and to collaborate with adult providers to "provide support and resources for transitioning young adults" (I6). Finally, the International Society of Pediatric and Adolescent Diabetes (ISPAD) guidelines additionally highlight that transition of care should be "a component of a highquality, multidisciplinary diabetes service ... and must involve both teams of carers, an understanding of the two different systems of care, and the differing expectations of those providing and those receiving care" (I5). Both AAP and ISPAD guidelines additionally address the potential need to delay transition of care for individuals with unique needs $(3,15)$.

According to recent data from the TID Exchange Clinic Registry in the United States, the average AIC for youth with type I diabetes is significantly above target, peaking at $9.3 \%$ in midadolescence, with only $17 \%$ of patients who are $<_{\mathrm{I}} 8$ years of age reaching the ADA goal of $<7.5 \%$ (I7). $\mathrm{AIC}$ is an important marker not only of metabolic health and thus risk of long-term complications of diabetes, but also of potential risk for acute complications. In $2019 \mathrm{TID}$ Exchange data, patients with an $\mathrm{AIC}>9.0 \%$ had a $7 \%$ incidence of diabetic ketoacidosis in the past 3 months, compared with a $0.7 \%$ incidence in patients with an $\mathrm{AIC}<8.0 \%$ (17). Of note, prior studies have also shown that AIC may rise after transition to adult care (I8,I9). Limited literature on this population suggests that it may be appropriate to delay transition of care until improvements in AIC are documented; however, this suggestion creates logistical difficulties for systems that attempt to transition patients based on age. Therefore, discussions about transitions of care for individuals with elevated pre-transition AIC levels contain an additional level of complexity, and little has been written about this specific cohort in the literature.

Qualitative research offers a unique opportunity to understand attitudes, perceptions, beliefs, and expectations of the target population (20). Multiple qualitative studies have explored expectations and experiences with pediatric and adult care before and after transition among young adults with type I diabetes, but the mean AIC values in these studies generally cluster around the target range, and the voices of patients with an AIC above goal are underrepresented (4,6,2I-24). Given that all adolescents and young adults, including those with an elevated $\mathrm{AIC}$, require tailored transition education and planning, developing an in-depth appreciation for their lived experience and perspectives is a crucially important step in improving transition care and outcomes for this at-risk population.

\section{Research Design and Methods}

\section{Study Design}

We conducted one-on-one semi-structured interviews of adolescents (aged I3-I7 years) and young adults (aged I8-23 years) with type I diabetes and an $\mathrm{AIC}>9 \%$ to elicit their perspectives pertaining to transition from pediatric to adult diabetes care. The institutional review board of Boston Children's Hospital approved this study.

\section{Sample}

We applied purposive sampling techniques to recruit adolescents and young adults (aged I4-23 years) who were diagnosed with diabetes $>_{\text {I }}$ year ago and who, at the time of the interviews, were receiving care at a comprehensive pediatric diabetes center operating within a referral pediatrics hospital in Boston, MA (20). At this pediatric diabetes center at the time of the study, there was no formal transition programing. To be eligible for our study, participants had to have an $\mathrm{AIC}>9.0 \%$ (measured via DCA Vantage Analyzer [Siemens Medical Solutions, Malvern, PA]). All I4 participants completed informed consent (or a parent/guardian consented and the participant gave assent, depending on age) and were compensated $\$ 50$ for their time.

\section{Interview Guide and Procedures}

Our research team created a detailed interview guide based on multidisciplinary expert consensus and an extensive review of the academic literature on transitions of care from pediatric to adult practice (see the Supplemental Material). Experts included the study senior author (K.G.), as well as 
other pediatric diabetologists, psychologists experienced in diabetes care and qualitative research, and adult diabetologists. Two members of the study team (T.B. and T.D.) conducted the $\mathrm{I} 4$ independent interviews in 20I6. Interviews were professionally transcribed.

Interview questions ranged from open-ended questions designed to elicit personal experiences and subjective responses to more precise, focused questions designed to collect a range of responses to the same question. Major topics covered in these interviews included patients' knowledge of the transition process, patients' perceptions of the appropriate age to begin the process, patients' perceptions of their independence and of their family's role in diabetes care, patients' experience of pediatric care, and patients' perceptions of adult care.

\section{Data Analysis}

Thematic analysis is an accepted and commonly used method in qualitative research, allowing investigators to consolidate data across multiple participants and generate over-arching conclusions $(25,26)$. Given the existing body of literature on this topic, we selected framework analysis for our specific approach (27). To begin the framework analysis procedures for this study, an initial code set was generated from extensive literature review of previous qualitative work in diabetes transition. Codes from this framework were applied to the transcripts; additionally, many novel codes were generated and added to the framework throughout the analysis process (27).

Three team members (E.S.T., J.R., and K.G.) independently read each interview transcript and coded it according to the aforementioned thematic analysis. The study team iteratively met to review individual coding and come to a consensus regarding all codes for each interview. During the progressive analysis of transcripts, saturation with regard to thematic content was clearly reached based on research team consensus. After the coding process, codes were entered into NVivo I2 software (QSR International Pty Ltd., Victoria, Australia) to enable further thematic grouping and analysis.

The team met to conduct second-level coding and ultimately identify final, key themes best representing the body of data. An audit trail was maintained to track the process of generating final themes.

\section{Results}

\section{Participant Characteristics}

The research team conducted interviews of I4 adolescents and young adults with type I diabetes and an

\begin{tabular}{lc} 
TABLE 1 Demographic Characteristics of Participants \\
$(n=14)$ \\
\hline Male & 57 \\
\hline Adolescent (<18 years of age) & 64 \\
\hline Young adult (18-23 years of age) & 36 \\
\hline Caucasian & 79 \\
\hline Hispanic & 14 \\
\hline Type 1 diabetes duration, years & $8.2 \pm 4.6$ \\
\hline Insulin pump use & 71 \\
\hline A1C, overall, \%* & $9.64 \pm 0.9$ \\
\hline A1C, adolescents, \%* & $9.73 \pm 1.0$ \\
\hline A1C young adults, \%* & $9.42 \pm 0.3$ \\
\hline
\end{tabular}

Data are percentages or mean $\pm \mathrm{SD}$. ${ }^{*} \mathrm{~A} 1 \mathrm{C}$ averaged over four most recent visits, including on the day of interview, if available.

AIC $>9 \%$ (Table I). Participants were between 14 and 23 years of age. Nine participants were adolescents (mean age $15.0 \pm 0.9$ years) and five were young adults (mean age $20.8 \pm 2.2$ years); $57 \%$ of the sample was male. Of the participants, $79 \%$ were Caucasian and $\mathrm{I} 4 \%$ identified as Hispanic.

Duration of diabetes ranged from 2 to 18 years, with a mean and SD of 8.2 and 4.6 years, respectively. Average AIC was $9.73 \pm$ I.0\% for adolescents and $9.42 \pm 0.3 \%$ for young adults who participated.

\section{Thematic Analysis}

Analysis of the I4 semi-structured interviews yielded the following four themes regarding transition from pediatric to adult care in teenagers with type I diabetes and an AIC >9\%: I) lack of formal preparation, 2) desire for delayed and gradual transition, 3) attachment to pediatric providers, and 4) concern about an impersonal adult care setting. Based on the research team's coding and subsequent thematic analysis, these themes were found to be consistent across adolescents and young adults. The following section provides more detailed explanations of the described themes, as well as illustrative quotations from interview transcripts.

\section{Theme 1: Lack of Formal Preparation}

Regardless of age and duration of diabetes, participants reported that they did not receive any formal transition preparation. They tended to believe it was the responsibility of the provider to bring up transition when appropriate and generally were unaware of what the process would entail. 
I think [the transition process] will all just come naturally.

(19-year-old female, diabetes duration 9 years)

I really have no concerns [about transitioning]. Like, it will be a smooth transition, I guess. (I6-year-old male, diabetes duration 3 years)

Participants consistently seemed surprised by the questions about transition and uncertain of the expectations of them or their providers. When they had considered the impending transition, the most they tended to expect from their providers was a list of referrals but had not further considered what they or their provider needed to do to prepare for the transition.

I haven't really thought, actually I have, I've thought about like, oh, what's going to happen when I'm older . . because I know that you can keep coming here until you're like I8 or something like that? (I5-year-old female, diabetes duration 9 years)

She gave me a list of doctors that I should look into that she thought would be helpful for me... . It was more just a list of names. (22-year-old male, diabetes duration II years)

\section{Theme 2: Desire for Delayed and Gradual Transition}

Both adolescents and young adults, regardless of their age, expressed the opinion that transition discussions and transition itself should happen in the future. Although the degree of delay of transition planning varied across participants, the desire for delay was essentially universal. The following are a series of responses interviewers received to questions about when it would be appropriate to think about transitioning to adult care.

... probably when my primary diabetes doctor, when he retires or stops practicing. (I9-year-old female, diabetes duration 9 years)

Probably mid-20s. (I9-year-old female, diabetes duration 9 years)

Umm, probably after high school .... Yeah. It's like, over I8, I guess. (I6-year-old male, diabetes duration 3 years)

Maybe like 25 . . . like you're living on your own ... you know, taxes and stuff like that, that's adult stuff. (I5-yearold female, diabetes duration 9 years)

Patients who were least inclined to plan for transition of care suggested changes in health insurance and/or major life transitions such as moving to college as the only real impetus to consider switching care to an adult provider and actively endorsed avoidance. They asserted a desire to "deal with" transition of care later, once other issues in their lives and health care were more settled.
So, I'd like to think by the time I'm 24 or 25 , I'll look into the transition. I'll start that process. But, umm, I would like to have everything kept under wraps before I turn 26, and [am] taken off my family health care plan, so I don't have to deal with too much change at once. (23-year-old male, diabetes duration 18 years)

I don't want to talk about it ... I just turned I8, so I don't want to leave yet. (I8-year-old female, diabetes duration 7 years)

MODERATOR: When do you think you would be ready to transition to an adult doctor? PARTICIPANT: I . . really don't know. Maybe like in my 30s. (I6-year-old female, diabetes duration 2 years)

Umm, maybe when I was like, maybe when I had my license maybe, or like when I was actually maybe moved out of my parents' house. That's probably when I would know I should probably change. (I4-year-old male patient, diabetes duration 8 years)

\section{Theme 3: Attachment to Pediatric Providers}

Interview participants demonstrated a near-universal sense of attachment to their pediatric care providers, which was often accompanied by a hesitancy to consider leaving their care.

I'm very comfortable with him, and I've been with him for almost Io years, so I would probably go to adult primary care after [he retires] just so I can, you know, end the relationship on a good note. (22-year-old male, diabetes duration II years)

I've been with the same doctors for a very long time so, you know, I feel very comfortable with them. (22-year-old male, diabetes duration 12 years)

Multiple participants focused on the notion that their pediatric providers knew their whole history and unique elements of their lives and diabetes.

I've been seeing my endocrinologist now for ... going on I2 years [and] she knows me very well on a personal and a diabetic level. (22-year-old male, diabetes duration II years)

... these people have been with me . . they know . . my state of mind. They know, like, all my struggles that I've been through. (I8-year-old female, diabetes duration 7 years)

Additionally, they describe pediatric care as more gentle and friendlier, which stands in contrast to their perceptions of adult care (see Theme 4: Concern About an Impersonal Adult Care Setting). 
My doctors now are very friendly . . very approachable, and easy to talk to. (I6-year-old male, diabetes duration 3 years)

I feel like it'd be a lot more formal. Like, my doctor cuts me a lot of slack sometimes.... She just tries to, like, ease you into doing what's right and stop doing the mistakes that you've been doing. I feel like it's a lot easier to talk to her that way than if it was an adult doctor. Then, he'll just tell you right away. (15-year-old male, diabetes duration 9 years)

\section{Theme 4: Concern About an Impersonal Adult Care Setting}

When asked about their concerns about transition and perceptions of adult care, participants had pervasive anxieties about switching to new providers and their ability to form therapeutic relationships.

I think it would be awkward, and it would be hard to, like, build a bond with them. (15-year-old female, diabetes duration 9 years)

Umm, my concern about it is, like, having doctors trying to just, like, dose me up, like I'm saying earlier. And, like, not understanding what's, like, my history, my background history. (16-year-old female, diabetes duration 2 years)

At least in part, these concerns seemed to be driven by a perception that adult care providers would be unable to meet young adult patients' needs and would instead be more direct and less lenient than their pediatric providers.

And I was more nervous that when I switch over out of pediatrics that they wouldn't be willing to ... see me that often, and that it might be a problem that I wouldn't be able to be on top of my diabetes as well. Because I've always kind of needed somebody else to just kind of be there. (22-year-old male, diabetes duration II years)

I just, I think it's going to be a lot scarier because you just get to a new level where he's going to be up front and just truthful with you, and, like, I don't know how I'm going to take that. (15-year-old male, diabetes duration 9 years)

They're probably going to treat me like an adult ... because here, they kind of ... sugarcoat things more than an adult endocrinologist would, and like, I feel if you go to an adult, then they're going to really talk about, like, the bad things that could happen if you don't care if your diabetes, like, you're ... it's going to really hit you harder. (15-year-old female, diabetes duration 9 years)

Given these concerns about their potential future adult care settings, participants voiced a desire for their current pediatric providers to share personalized lists of adult physicians as part of the transition process.

Yeah. Like a rec, like doctors ... that they'd recommend, that they'd know, like, I'd be successful with, I guess. (I6-yearold male, diabetes duration 3 years)

Umm, in my ideal world ... my doctor will suggest a person she knows can give good care and that she has a personal relationship with so I'm not, I'm going to someone with the same type of philosophy as her. So, I'm hoping that she makes her recommendation as I get closer. (23-year-old male, diabetes duration $\mathrm{I} 8$ years)

Giving references on what they think should be helpful for me.... I just feel like I would want to hear what they had to say about, their opinion on kind of what doctor would lean toward, like, my situation because I've been through 7 years, have been rough for me, so like . . (18-year-old female, diabetes duration 7 years)

\section{Discussion}

This qualitative study detailed the voices and perspectives on transition to adult care of adolescents and young adults with type I diabetes and an $\mathrm{AIC}>9 \%$ who were cared for at a U.S. pediatric center. The semi-structured interview format elicited patients' thoughts on a range of transitionrelated topics. Rigorous thematic analysis allowed for the generation of clear themes regarding their shared experiences and opinions. Notably, these data shed light on a vulnerable population previously underrepresented in the literature, and our findings provide guidance for pediatric providers in prioritizing topics for discussion with this population.

The logic of qualitative sampling rests on the idea of transferability (i.e., whether the results advance theoretical understandings and are relevant to multiple situations) rather than generalizability or representativeness (28). The themes that emerged from this analysis focus primarily on ignorance about transition, desire to delay transition and remain comfortable in pediatric provider relationships, and anxiety about a stricter adult care setting.

The mean AIC of this study population was $9.64 \%$, which more closely mirrors national averages in this demographic compared with past studies that have relied on less representative samples with AIC levels closer to ADA targets $(4,7,17,18,21,22,29)$. For example, in a large national survey of transition experiences, $78 \%$ of patients had a self-reported $\mathrm{AIC}<9.0 \%(7)$. 
Previous studies (in populations with relatively lower AIC levels) showed deficits in transition preparation counseling $(6,7, \mathrm{II}, 22)$. Here, we provide detailed perspectives from a population of adolescents and young adults with type I diabetes and an $\mathrm{AIC}>9 \%$ voicing a similar lack of awareness or understanding about the transition process. It should be noted that, at the time of this study, the center at which these patients received their care did not have any formal transition programming in place. Beyond this, however, we show a new theme spanning the age range of this study's participants: the desire of both teens and young adults to defer transition and "deal with it later." This tendency toward deferring transition preparation is informative for providers as they consider how to guide this population through a safe and appropriately timed transition.

In general, lack of transition preparation has been associated with gaps between pediatric and adult care, which in turn may contribute to poor outcomes $(4,7)$. This patient population is already at high risk of both acute and chronic complications because of elevated AIC, making the reported lack of transition preparation among this vulnerable subset of patients with type I diabetes particularly concerning for providers $(29,30)$.

Although our study did specifically elicit provider perspectives, providers may be more likely to defer transition conversations with patients who have higher $\mathrm{AIC}$ values (i.e., wait for a period in which target glucose levels are met). A U.S. national survey of pediatric endocrinologists caring for patients with AIC levels above target raised this issue, although findings were inconsistent in terms of the precise relationship between the decision to transition patients and AIC level (3I). This topic warrants further research, and diabetes care providers may benefit from enhanced further consideration and guidelines regarding the role of AIC level in determining transition timing and disposition.

When prompted to consider the transition preparation components that may be most useful, it is interesting to note that teens with elevated AIC recognize the value of referrals to recommended providers and even the idea of communication between adult and pediatric providers, as has been noted in past studies (2I,22). This recommendation is iteratively represented in all diabetes transition consensus statements and is explored in interventional work; however, it has yet to translate consistently into practice $(5,32)$.

Surveys of both pediatric providers and patients have demonstrated the important issue of mutual attachment between pediatric providers and patients with type I diabetes (7,3I). Previous qualitative work in post-transition young adults suggested increased attachment and reticence to transition among patients with target $\mathrm{AIC}$ compared with those with AIC above target, who wanted a "fresh start" (33). Our sample of pediatric patients voiced high levels of attachment to their providers, which served as a hindrance to considering transition. The pervasive nature of this sentiment suggests the need to develop strategies to openly address attachment and facilitate the emotional aspects of transition for patients across the AIC spectrum.

Along similar lines, our findings highlight a need for pediatric providers to explicitly address preconceived notions about adult diabetes care. Perceptions of adult care as being franker and more focused on long-term diabetes complications have been borne out in other qualitative literature on the subject $(7,22)$. However, our cohort focused particularly on a desire to be known by their new provider, which must also be addressed in preparing for transition.

This study has a number of limitations. First, the patient population reflects that of a specialty pediatric diabetes center in a referral pediatric hospital and, as such, does not appropriately represent the diversity of patient experiences across the United States or internationally. However, qualitative research is not designed to create a representative sample per se, but rather to understand different perspectives (28). Further research should be conducted in different patient demographic groups, as well as with providers and families, to expand our understanding of transition perspectives among patients with a broader spectrum of glycemic status and among their caregivers. Additional research of a more quantitative nature would also enhance our understanding of the risks and benefits of transition at particular ages for patients with elevated AIC. A prospective cohort study of glucose levels across the transition in different care delivery models would also further advance the field.

\section{Conclusion}

This qualitative study generated several important themes regarding the perspectives of adolescents and young adults with type I diabetes and an $\mathrm{AIC}>9 \%$ on transition to adult care. Their lack of preparation for transition, desire to delay the process, attachment to their pediatric providers, and fear/concerns about adult care suggest a number of areas for potential intervention. Explicit conversations must take place to make youth aware of identified issues in the transition to adult care. Shared decision-making and planning cannot be deferred, even if transition itself is appropriately delayed. Given the uniquely vulnerable position of these patients, further research is warranted to 
generate and test interventions to protect their short- and long-term health during the transition period and beyond.

\section{FUNDING}

This project was supported by National Institute of Diabetes and Digestive and Kidney Disease grant NIDDK K23DK102655. E.S.T. was supported by grant number T32-HS000063 from the Agency for Healthcare Research and Quality. The content is solely the responsibility of the authors and does not necessarily represent the official views of the Agency for Healthcare Research and Quality. E.S.T. was also supported by National Institutes of Health grant T32-DK007699.

\section{DUALITY OF INTEREST}

No potential conflicts of interest relevant to this article were reported.

\section{AUTHOR CONTRIBUTIONS}

E.S.T. assisted in coding the interview transcripts and generating themes and wrote the manuscript. J.R. assisted in coding the interview transcripts, generating themes, and writing and revising the manuscript. T.B. and T.D. recruited and interviewed participants. K.G. conceptualized the study design, created the interview guide, and assisted in coding the transcripts, generating themes, and writing and revising the manuscript. K.G. is the guarantor of this work and, as such, had full access to all the data in the study and takes responsibility for the integrity of the data and the accuracy of the data analysis.

\section{PRIOR PUBLICATION}

This project was previously presented in poster form at the 45th annual conference of the International Society of Pediatric and Adolescent Diabetes, 30 October to 2 November 2019, in Boston, MA.

\section{REFERENCES}

1. Blum RW, Garell D, Hodgman $\mathrm{CH}$, et al. Transition from childcentered to adult health-care systems for adolescents with chronic conditions: a position paper of the Society for Adolescent Medicine. J Adolesc Health 1993;14:570-576

2. Arnett JJ. Emerging adulthood: a theory of development from the late teens through the twenties. Am Psychol 2000;55:469-480

3. White PH, Cooley WC; Transitions Clinical Report Authoring Group; American Acedemy of Pediatrics; American Academy of Family Physicians; American College of Physicians. Supporting the health care transition from adolescence to adulthood in the medical home. Pediatrics 2018;142:e20182587

4. Busse FP, Hiermann P, Galler A, et al. Evaluation of patients' opinion and metabolic control after transfer of young adults with type 1 diabetes from a pediatric diabetes clinic to adult care. Horm Res 2007;67:132-138

5. Nakhla M, Daneman D, To T, Paradis G, Guttmann A. Transition to adult care for youths with diabetes mellitus: findings from a Universal Health Care System. Pediatrics 2009;124:e1134-e1141

6. Garvey KC, Wolpert HA, Rhodes ET, et al. Health care transition in patients with type 1 diabetes: young adult experiences and relationship to glycemic control. Diabetes Care 2012;35:1716-1722

7. Garvey KC, Foster NC, Agarwal S, et al. Health care transition preparation and experiences in a U.S. national sample of young adults with type 1 diabetes. Diabetes Care 2017;40: 317-324

8. Sandler CN, Garvey KC. A practice in maturation: current perspectives on the transition from pediatric to adult care for young adults with diabetes. Curr Diab Rep 2019;19:126

9. Sezgin E, Weiler M, Weiler A, Lin S, Hart L. It is a life journey: a roadmap of teens with chronic diseases in transitioning to independence. J Pediatr Health Care. Epub ahead of print on 11 March 2020 (DOI: 10.1016/j.pedhc.2020.02.001)
10. Colver A, Rapley T, Parr JR, et al. Facilitating transition of young people with long-term health conditions from children's to adults' healthcare services: implications of a 5-year research programme. Clin Med (Lond) 2020;20:74-80

11. Iversen $\mathrm{E}$, Kolltveit $\mathrm{BH}$, Hernar I, Mårtensson J, Haugstvedt $\mathrm{A}$. Transition from paediatric to adult care: a qualitative study of the experiences of young adults with type 1 diabetes. Scand J Caring Sci 2019;33:723-730

12. Goethals ER, Commissariat PV, Volkening LK, Markowitz JT, Laffel LM. Assessing readiness for independent self-care in adolescents with type 1 diabetes: introducing the RISQ. Diabetes Res Clin Pract 2020;162:108110

13. Pierce J, Aroian K, Schifano E, Gannon A, Wysocki T. Development and content validation of the Healthcare Transition Outcomes Inventory for young adults with type 1 diabetes. J Patient Rep Outcomes 2019;3:71

14. Peters A, Laffel L, Albright A, et al.; American Diabetes Association Transitions Working Group. Diabetes care for emerging adults: recommendations for transition from pediatric to adult diabetes care systems. Diabetes Care 2011;34:2477-2485

15. Cameron FJ, Garvey K, Hood KK, Acerini CL, Codner E. ISPAD clinical practice consensus guidelines 2018: diabetes in adolescence. Pediatr Diabetes 2018;19(Suppl. 27):250261

16. American Diabetes Association. 13. Children and adolescents: Standards of Medical Care in Diabetes-2019. Diabetes Care 2019;42(Suppl. 1):S148-S164

17. Foster NC, Beck RW, Miller KM, et al. State of type 1 diabetes management and outcomes from the T1D Exchange in 2016-2018. Diabetes Technol Ther 2019;21:66-72

18. Helgeson VS, Reynolds KA, Snyder PR, et al. Characterizing the transition from paediatric to adult care among emerging adults with type 1 diabetes. Diabet Med 2013;30:610-615

19. Lotstein DS, Seid M, Klingensmith G, et al.; SEARCH for Diabetes in Youth Study Group. Transition from pediatric to adult care for youth diagnosed with type 1 diabetes in adolescence. Pediatrics 2013;131:e1062-e1070

20. Rice P, Ezzy D. Qualitative Research Methods: A Health Focus. Oxford, U.K., Oxford University Press, 1999

21. Garvey KC, Beste MG, Luff D, Atakov-Castillo A, Wolpert HA, Ritholz $M D$. Experiences of health care transition voiced by young adults with type 1 diabetes: a qualitative study. Adolesc Health Med Ther 2014;5:191-198

22. Hilliard ME, Perlus JG, Clark LM, et al. Perspectives from before and after the pediatric to adult care transition: a mixed-methods study in type 1 diabetes. Diabetes Care 2014;37:346-354

23. Dovey-Pearce G, Hurrell R, May C, Walker C, Doherty Y. Young adults' (16-25 years) suggestions for providing developmentally appropriate diabetes services: a qualitative study. Health Soc Care Community 2005;13:409-419

24. Gee L, Smith TL, Solomon M, Quinn MT, Lipton RB. The clinical, psychosocial, and socioeconomic concerns of urban youth living with diabetes. Public Health Nurs 2007;24:318-328

25. Boyatzis R. Transforming Qualitative Information: Thematic Analysis and Code Development. Thousand Oaks, CA, Sage Publications, 1998

26. Braun V, Clarke V. Using thematic analysis in psychology. Qual Res Psychol 2008;206:77-101

27. Gale NK, Heath G, Cameron E, Rashid S, Redwood S. Using the framework method for the analysis of qualitative data in multidisciplinary health research. BMC Med Res Methodol 2013;13:117

28. Kuper A, Reeves S, Levinson W. An introduction to reading and appraising qualitative research. BMJ 2008;337:a288 
29. Garvey KC, Wolpert HA, Laffel LM, Rhodes ET, Wolfsdorf JI, Finkelstein JA. Health care transition in young adults with type 1 diabetes: barriers to timely establishment of adult diabetes care. Endocr Pract 2013;19:946-952

30. Jacobson AM, Hauser ST, Willett J, Wolfsdorf JI, Herman L. Consequences of irregular versus continuous medical follow-up in children and adolescents with insulin-dependent diabetes mellitus. J Pediatr 1997;131:727-733

31. Agarwal S, Garvey KC, Raymond JK, Schutta MH. Perspectives on care for young adults with type 1 diabetes transitioning from pediatric to adult health systems: a national survey of pediatric endocrinologists. Pediatr Diabetes 2017;18:524531

32. Spaic T, Robinson T, Goldbloom E, et al.; JDRF Canadian Clinical Trial CCTN1102 Study Group. Closing the gap: results of the multicenter Canadian randomized controlled trial of structured transition in young adults with type 1 diabetes. Diabetes Care 2019; 42:1018-1026

33. Ritholz MD, Wolpert H, Beste M, Atakov-Castillo A, Luff D, Garvey $\mathrm{KC}$. Patient-provider relationships across the transition from pediatric to adult diabetes care: a qualitative study. Diabetes Educ 2014;40:40-47 